\title{
Performance Analysis of V-Blast Based MIMO-OFDM System with Various Detection Techniques
}

\author{
Dr. Anubhuti Khare ${ }^{1}$, Manish Saxena ${ }^{2}$, Vijendra singh mandloi ${ }^{3}$ \\ 1. Dr. Anubhuti Khare, Reader, Department of Electronics and Communication, University Institute \\ Of Technology, Rajeev Gandhi Technical University, Bhopal. \\ 2. Manish Saxena, Head of Electronics and Communication Department, Bansal Institute Of Science \\ And Technology Bhopal. \\ $3 *$ Vijendra singh mandloi, Student, Mtech (Digital Communication), Bansal Institute of Science \\ And Technology Bhopal.
}

\begin{abstract}
-
This paper presents the performance analysis of $\mathrm{V}$ BLAST based multiple input multiple output orthogonal frequency division multiplexing (MIMOOFDM) system with respect to bit error rate per signal to noise ratio (BER/SNR) for various detection techniques viz zero forcing $(\mathrm{ZF})$, minimum mean square error (MMSE) and maximum likelihood (ML). A 2X2 MIMO-OFDM system is used for the performance evaluation. The simulation results shows that the performance of V-BLAST based detection techniques is much better than the conventional methods.

Keywords- Vertical Bell Labs Layered SpaceTime (V-BLAST); multiple input multiple output (MIMO); orthogonal frequency division multiplexing $(O F D M)$; bit error rate (BER); signal to noise ratio (SNR).
\end{abstract}

\section{INTRODUCTION}

With the increasing demand for high speed wireless transmission of data, the demand for vast frequency spectrum arises. Since the available frequency spectrum is scarce, future systems should be characterized by significantly enhanced spectral efficiency in order to increase link throughput and network capacity. By using multiple antennas at both transmitter and receiver (MIMO system) the throughput can be increased by simultaneously transmitting different streams of data on the different transmit antennas but at the same carrier frequency [1]. These parallel streams of data can be recovered at the transmitter with spatial sampling and corresponding signal process algorithm [2]. The combination of the throughput enhancement of MIMO system with the robustness of orthogonal frequency division multiplexing (OFDM) against frequency-selective fading caused by severe multipath scattering and narrowband interference is regarded as a very promising basis for future high

speed data communication. That's why there is high demand for a MIMO-OFDM system with high efficiency and low complexity.
Among conventional detection techniques in MIMO-OFDM communication such as zero forcing (ZF) [3], minimum mean square error (MMSE) [4] and maximum likelihood (ML) [5], ML shows better performance at the cost of higher complexity [6]. Therefore we have applied V-BLAST algorithm on all of the above detection techniques and then compared their performance on the basis of bit error rate per signal to noise ratio and found that the detection techniques with V-BLAST algorithm shows better performance than without V-BLAST. Even the complexity of the system reduces by using V-BLAST algorithm and the simulation results show that the performance of V-BLAST based MMSE is close to that of ML technique, with much lower complexity. Therefore V-BLAST based detection techniques can be preferred over conventional methods in MIMOOFDM system with high data transmission capacity where high efficiency is required with lower complexity. The analysis presented in this paper shows the performance comparison of conventional detection techniques in MIMO-OFDM communication with that of V-BLAST based techniques and hence shows that better performance can be achieved by using V-BLAST based detection techniques.

The remaining paper is organized as follows. In section II we present the V-BLAST based system model. Section III presents the performance comparison of various detection techniques with their V-BLAST counterpart. Simulation results are presented in section IV followed by the conclusions in section $\mathrm{V}$.

\section{V-BLAST BASED SYSTEM MODEL}

A V-BLAST based 2X2 MIMO-OFDM system is presented in figure (1). We considered $\mathrm{N}_{\mathrm{T}}$ transmitting antennas and $\mathrm{N}_{\mathrm{R}}$ receiving antennas. The incoming signal bits are multiplexed and then modulated with BPSK modulation [7]. The modulated signal is processed through inverse discreet Fourier transform (IDFT) to insert a cyclic prefix and convert it to a radio frequency and then transmit through channel [8]. 


\section{Dr. Anubhuti Khare, Manish Saxena, Vijendra singh mandloi / IOSR Journal of Engineering (IOSRJEN)

Vol. 2 Issue 1, Jan.2012, pp.166-169

At the receiver end, the signal is received through $\mathrm{N}_{R}$ antennas and processed through discreet Fourier

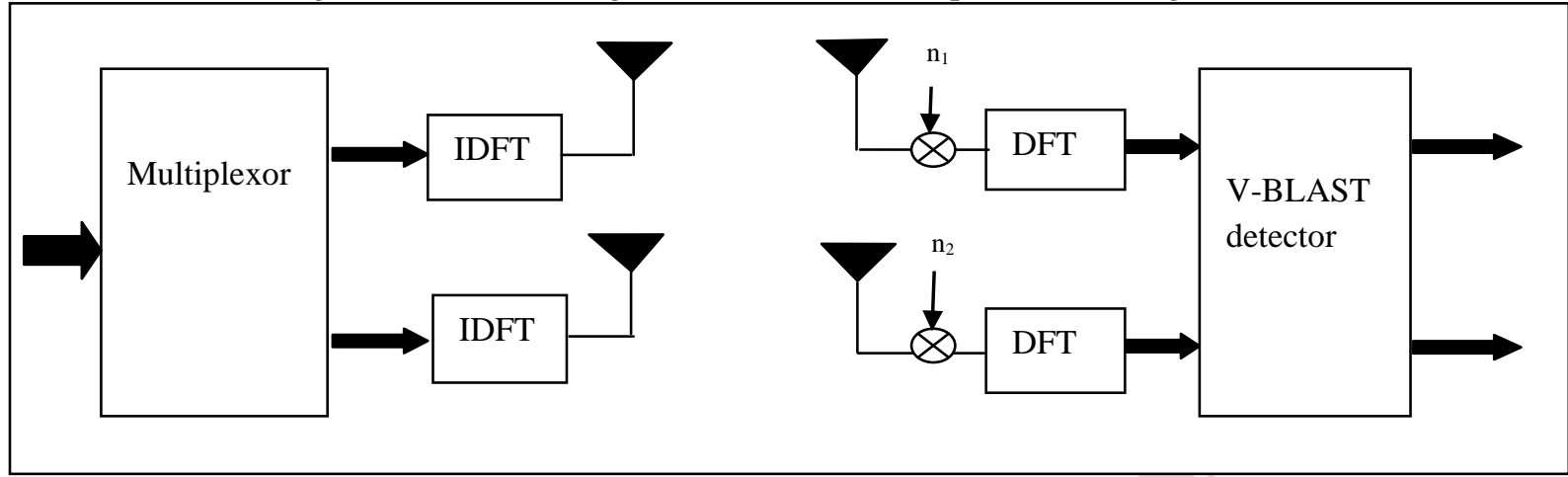

Figure (1): A 2X2 MIMO OFDM system with V-BLAST detector

transform (DFT) and then passed through V-BLAST detector. The received signal is passed through DFT to convert it to frequency domain. Hence the equation of received signal at first antenna is,

$$
y_{1}=\left[h_{1,1} h_{1,2}\right]\left[\begin{array}{l}
x_{1} \\
x_{2}
\end{array}\right]+n_{1}
$$

and the received signal at the second receive antenna will be,

$$
y_{2}=\left[h_{2,1} h_{2,2}\right]\left[\begin{array}{l}
x_{1} \\
x_{2}
\end{array}\right]+n_{2}
$$

So, the equivalent equation for the received signal can be written as,

$$
y=H x+n
$$

where $\mathrm{x}$ denotes the transmitted signal and $\mathrm{n}$ denotes the additive white Gaussian noise(AWGN) added to the signal.

The wideband MIMO channel is converted into a set of $\mathrm{N}$ parallel narrow band channels from (3). Now the V-BLAST algorithm is applied per subcarrier basis [9]. In V-BLAST a filter matrix $\mathrm{G}$ is multiplied to the signal and then detected layer by layer. The channel with maximum SNR is then selected and the effect of channel is nullified for that stream. Now the nullified symbols are quantized with appropriate constellation and the effect of detected stream is cancelled from the received signal. A new channel matrix is created then by replacing the columns corresponding to the detected stream with zeros and the whole process is repeated with the new channel matrix until all the streams are detected.

The algorithm for the whole process can be given as,

Finding nulling solution:

$$
\boldsymbol{G}=\left(\begin{array}{ll}
g_{11} & g_{12} \\
g_{21} & g_{22}
\end{array}\right)
$$

Ordering:

$$
\rho=\left\{\left\|(\boldsymbol{G})_{1}\right\|^{2},\left\|(\boldsymbol{G})_{2}\right\|^{2}\right\}
$$

$$
k=\min (\rho)
$$

Nulling: Assuming $\mathrm{k}=2$, then the nullified vector $\mathrm{g}$ can be written as,

$$
\boldsymbol{g}=\left(\begin{array}{l}
g_{21} \\
g_{22}
\end{array}\right)
$$

Nullifying the effect of channel from signal,

$$
\mathrm{y}_{3}=g^{T} r
$$

Quantizing:

$$
x_{2}=\operatorname{Quant}\left(y_{2}\right)
$$

Cancelling: Cancel the effect of detected stream from the received signal,

$$
\boldsymbol{r}=\boldsymbol{r}-\left(\begin{array}{l}
h_{21} \\
h_{22}
\end{array}\right) x_{2}
$$

Finding new channel matrix:

$$
\boldsymbol{H}=\left(\begin{array}{ll}
h_{11} & 0 \\
h_{12} & 0
\end{array}\right)
$$

Iteration: Now repeat from step 1 to get the first stream.

\section{COMPARISON}

\section{A. Zero Forcing}

Zero Forcing is a linear detection technique. The pseudo inverse of the signal is applied to the received signal in order to make a decision about one user. So the equation for filter matrix $G$ for zero forcing will be,

$$
\boldsymbol{G}=\left(\boldsymbol{H}^{H} \boldsymbol{H}\right)^{-1} \boldsymbol{H}^{H}
$$

In this way the received signal is detected by zero forcing detectors. If V-BLAST algorithm is applied on ZF detector, equation 4 will be applied on ZF filter matrix. Figure 2 shows the performance comparison of ZF and ZF-V-BLAST. As it is evident from the figure, ZF with V-BLAST shows better performance in comparison to normal ZF in terms of BER/SNR. 


\section{Dr. Anubhuti Khare, Manish Saxena, Vijendra singh mandloi / IOSR Journal of Engineering}

(IOSRJEN)

\section{Vol. 2 Issue 1, Jan.2012, pp.166-169}

B.

\section{Minimum Mean Square Error}

MMSE is also a linear detection technique but more reliable than ZF in case of noisy channel [10]. MMSE does not apply pseudo inverse of signal to make decision about one user, instead it attenuates them to noise level thereby reducing the diversity order. From [4] the filter matrix for MMSE is,

$$
\boldsymbol{G}=\left(\boldsymbol{H}^{H} \boldsymbol{H}+\frac{N_{t}}{S N R} I_{N_{r}}\right)^{-1} \boldsymbol{H}^{H}
$$

Now V-BLAST algorithm can be applied over above filter matrix and results can be generated. Figure 3 shows the performance comparison of MMSE detector and MMSE-V-BLAST detector in terms of BER/SNR.

\section{Maximum Likelihood}

ML is a non-linear detection technique. The BER/SNR results of ML are better than MMSE detector but at the cost of additional complexity [6]. So ML is used in applications where high efficiency is requires. Now if we apply V-BLAST algorithm on ML, the performance will be better than ML detector which is clear from figure (4).

\section{SIMULATION RESULTS}

We have considered a 2X2 MIMO-OFDM channel with BPSK modulation and various detection techniques are applied along with V-BLAST algorithm and their performance is compared on the basis of bit error rate per signal to noise ratio (BER/SNR). The simulation result shows that when the detection techniques are applied with V-BLAST, they show better results even for higher values of SNR. Figure (5) shows the performance comparison of all detection techniques along with V-BLAST. As it is evident from the figure, we can obtain close to ML performance with less complexity by applying VBLAST algorithm on linear detection techniques and also if we require a system with performance better than ML then we can also apply V-BLAST on ML.

\section{CONCLUSION}

We have analyzed the performance of V-BLAST on various detection techniques and compared them on the basis of BER/SNR and hence obtained better performance without additional complexity. We can also evaluate which system can be best suited for a particular application on the basis of given comparison. We can also compare the given techniques on the basis of other parameters such as symbol error rate (SER), computational time and system configuration to obtain enhanced results.

\section{ACKNOWLEDGEMENT}

Mrs. Anubhuti Khare one of the authors is indebted to Director UIT RGPV Bhopal for giving permission for sending the paper to journal. Manish Saxena is also thankful to the Chairmen, Bansal Institute of Science $\&$ Technology Bhopal for giving permission to send the paper for publication. Last but not least Vijendra Singh Mandloi is also thankful to the HOD Mr. Manish Saxena Sir \& Chairmen of BIST Bhopal for giving permission to send the paper for publication.

\section{REFERENCES}

[1] S. Cui, A. J. Goldsmith, and A. Bahia (August, 2004). "Energy-efficiency of MIMO and Cooperative MIMO in Sensor Networks". IEEE journal Select. Areas of Communication. 22 (6): 1089-1098.

[2] Sam P. Alex and Lousy M.A. Jalloul, "Performance Evaluation of MIMO in IEEE802.16e/WiMAX", IEEE J. of Selected Topics in Signal Processing, VOLUME 2, NUMBER 2, April, 2008.

[3] R. Xu and F. C. M. Lau, "Performance analysis for MIMO systems using zero forcing detector over fading channels," IEE Proc. Communications, vol. 153 , no. 1,2 , pp. $74-80$, February 2006.

[4] Al-Dhahir, "FIR Channel-Shortening Equalizers for MIMO ISI Channels", IEEE Trans. Communication vol. 49, pp.213-218, Feb. 2001.

[5] Inkyu Lee and John M. Cioffi, "Design of Equalized Maximum-Likelihood Receiver", IEEE Communications Letters, VOLUME 2, No. 1, January 1998.

[6] Xu Zhu and Ross D. Murch,” Performance Analysis of Maximum Likelihood Detection in a MIMO Antenna System", IEEE Transactions on Communications, VOLUME 50, NUMBER 2, FEBRUARY 2002.

[7] Proakis, John G. (1995). Digital Communications. Singapore: McGraw Hill. ISBN 0-07-113814-5.

[8] B. Yang, Z. Cao, and K. Letaief, "Analysis of Low-Complexity Windowed DFT-Based MMSE Channel Estimation for OFDM Systems," IEEE Trans. Communication., VOLUME 49, pp. 1977-1987, Nov. 2001.

[9] W. Y an, S. Sun and Z. Lei, "A low complexity VBLAST OFDM detection algorithm for wireless LAN systems," IEEE Communication. Letter, VOLUME 8 , pp. 347-376, June 2004.

[10] H. V. Poor and S. Verdu, Probability of error in MMSE multiuser detection," IEEE Transactions on Information Theory, VOLUME 43, pp. 858871, May 1997. 
Dr. Anubhuti Khare, Manish Saxena, Vijendra singh mandloi / IOSR Journal of Engineering (IOSRJEN)

www.iosrjen.org

ISSN : 2250-3021

Vol. 2 Issue 1, Jan.2012, pp.166-169

TABLE I BER/SNR VALUES FOR VARIOUS DETECTION TECHNIQUES

\begin{tabular}{|c|c|c|c|c|c|}
\hline BER/SNR & \multicolumn{5}{|c|}{ SNR } \\
\hline ZF & 0.3250 & 0.1400 & 0.0420 & 0.0110 & 0.0020 \\
\hline ZF-VBLAST & 0.1470 & 0.0670 & 0.0140 & 0.0100 & 0.0010 \\
\hline MMSE & 0.2370 & 0.0950 & 0.0210 & 0.0040 & 0.0000 \\
\hline MMSE-VBLAST & 0.1070 & 0.0230 & 0.0100 & 0.0020 & 0.0000 \\
\hline ML & 0.2160 & 0.0490 & 0.0050 & 0.0000 & 0.0000 \\
\hline ML-VBLAST & 0.0600 & 0.0110 & 0.0000 & 0.0000 & 0.0000 \\
\hline
\end{tabular}

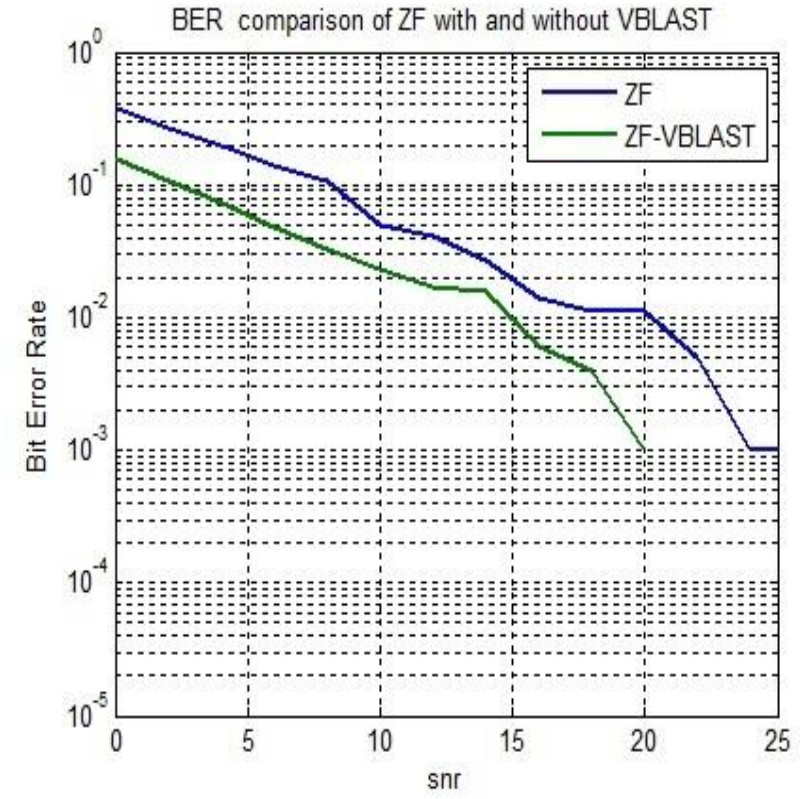

Figure (2): BER/SNR plot for ZF and ZF-V-BLAST

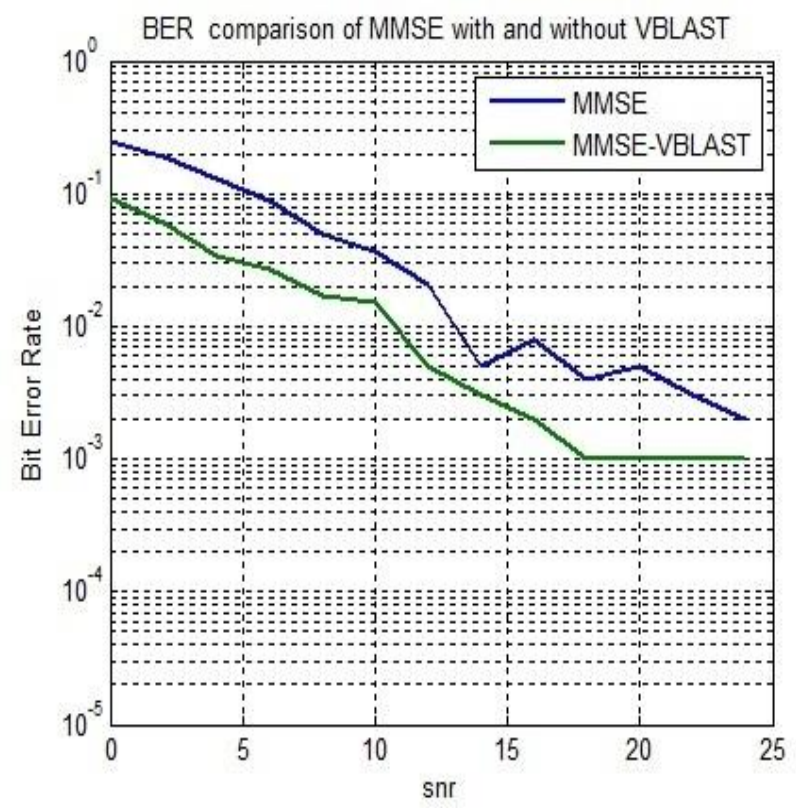

Figure (3): BER/SNR plot for MMSE and MMSE-V-BLAST

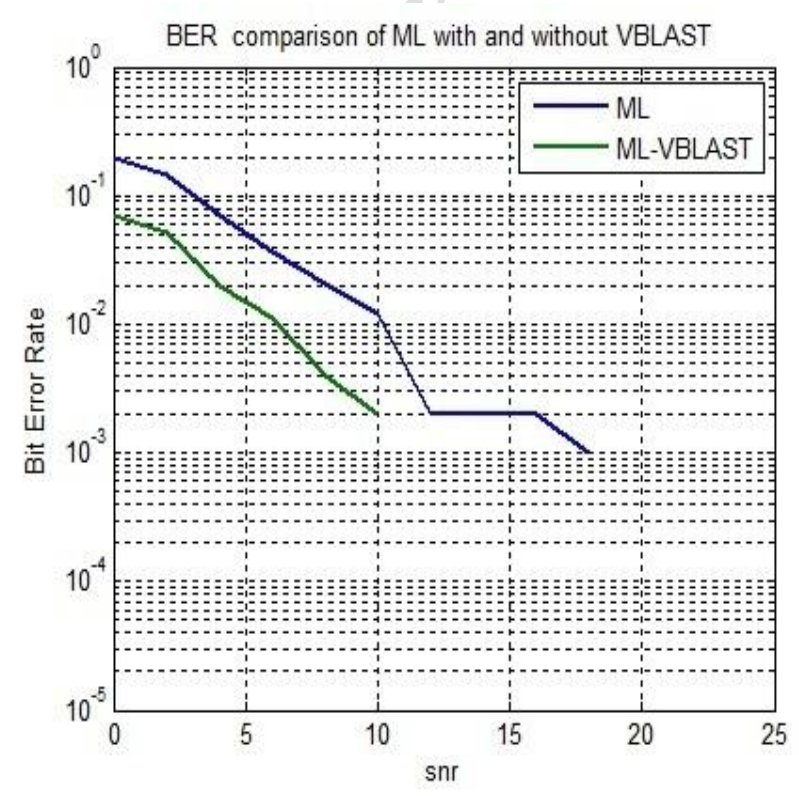

Figure (4): BER/SNR plot for ML and ML-VBLAST

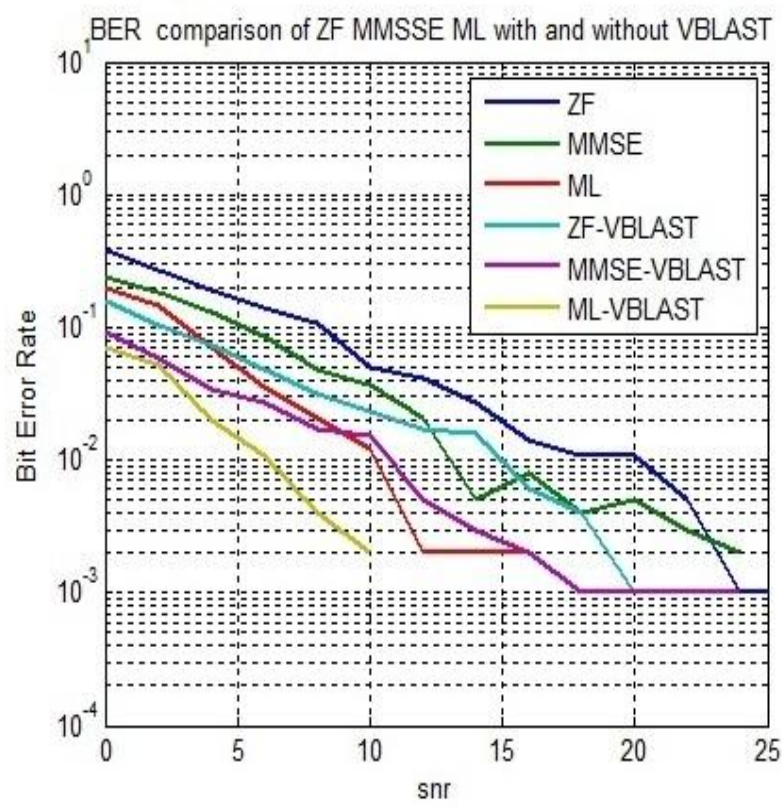

Figure (5): BER/SNR plot for all detection techniques. 